\title{
The growth and value distribution of Laplace-Stieltjes transformations with infinite order in the right half-plane
}

Hong-Yan Xu' and Zu-Xing Xuan²*

\section{"Correspondence:}

xuanzuxing@ss.buaa.edu.cn

${ }^{2}$ Beijing Key Laboratory of

Information Service Engineering,

Department of General Education,

Beijing Union University, No. 97 Bei

Si Huan Dong Road, Chaoyang

District, Beijing, 100101, China

Full list of author information is

available at the end of the article

\begin{abstract}
By introducing the concept of $X_{U}$-order functions, we study the growth of analytic functions defined by Laplace-Stieltjes transformations which converge on the right half-plane. Some necessary and sufficient conditions on finite $X_{U}$-order of these functions have been obtained. We also investigate the value distribution of Laplace-Stieltjes transformations with finite $X_{U}$-order and obtain the existence of $X_{U}$-points and $X_{U}$-points dealing with multiple values of two Laplace-Stieltjes transformations which converge on the right half-plane. The main results of this paper are improvement and extension of some theorems given by Shang and Gao.
\end{abstract}

MSC: 44A10; 30D15

Keywords: $X$-order; $X_{U}$-order; Laplace-Stieltjes transform

\section{Introduction and basic notes}

Consider the Laplace-Stieltjes transforms

$$
F(s)=\int_{0}^{+\infty} e^{-s x} d \alpha(x), \quad s=\sigma+i t,
$$

where $\alpha(x)$ is a bounded variation on any interval [0,Y] $(0<Y<+\infty)$, and $\sigma$ and $t$ are two real variables. We choose a sequence $\left\{\lambda_{n}\right\}_{n=1}^{\infty}$

$$
0=\lambda_{1}<\lambda_{2}<\lambda_{3}<\cdots<\lambda_{n} \uparrow+\infty
$$

which satisfies the following conditions:

$$
\begin{aligned}
& \limsup _{n \rightarrow+\infty}\left(\lambda_{n+1}-\lambda_{n}\right)<+\infty, \quad \limsup _{n \rightarrow+\infty} \frac{n}{\lambda_{n}}=D<\infty, \\
& \limsup _{n \rightarrow+\infty} \frac{\log A_{n}^{*}}{\lambda_{n}}=0,
\end{aligned}
$$

where

$$
A_{n}^{*}=\sup _{\lambda_{n}<x \leq \lambda_{n+1},-\infty<t<+\infty}\left|\int_{\lambda_{n}}^{x} e^{-i t y} d \alpha(y)\right| .
$$

( ) 2013 Xu and Xuan; licensee Springer. This is an Open Access article distributed under the terms of the Creative Commons Attribution License (http://creativecommons.org/licenses/by/2.0), which permits unrestricted use, distribution, and reproduction in any medium, provided the original work is properly cited. 
Remark 1.1 The Dirichlet series is regarded as a special example of Laplace-Stieltjes transformations, and considerable attention has been paid to the growth and the value distribution of analytic functions defined by the Dirichlet series; see [1-3] for some recent results.

In 1963, Yu [4] proved the Valiron-Knopp-Bohr formula of the associated abscissas of bounded convergence, absolute convergence and uniform convergence of LaplaceStieltjes.

Theorem A Suppose that Laplace-Stieltjes transformations (1) satisfy the first formula of (3) and $\lim \sup _{n \rightarrow+\infty} \frac{\log n}{\lambda_{n}}<+\infty$. Then

$$
\limsup _{n \rightarrow+\infty} \frac{\log A_{n}^{*}}{\lambda_{n}} \leq \sigma_{u}^{F} \leq \limsup _{n \rightarrow+\infty} \frac{\log A_{n}^{*}}{\lambda_{n}}+\limsup _{n \rightarrow+\infty} \frac{\log n}{\lambda_{n}}
$$

where $\sigma_{u}^{F}$ is called the abscissa of uniformly convergent $F(s)$.

It follows from (3), (4) and Theorem A that $\sigma_{u}^{F}=0$, i.e., $F(s)$ is analytic in the right halfplane. Put

$$
\begin{aligned}
& \mu(\sigma, F)=\max _{n \in N}\left\{A_{n}^{*} e^{-\lambda_{n} \sigma}\right\} \quad(\sigma>0), \quad M(\sigma, F)=\sup _{-\infty<t<+\infty}|F(\sigma+i t)|, \\
& M_{u}(\sigma, F)=\sup _{0<x<+\infty,-\infty<t<+\infty}\left|\int_{0}^{x} e^{-(\sigma+i t) y} d \alpha(y)\right| \quad(\sigma>0) .
\end{aligned}
$$

Remark 1.2 The concepts of $M_{u}(\sigma, F), \mu(\sigma, F)$ of analytic functions represented by Laplace-Stieltjes transformations convergent in the complex plane were first introduced by Yu.

Remark 1.3 From (4), for any $\sigma>0$, we have

$$
\limsup _{n \rightarrow+\infty} \frac{\log A_{n}^{*}-\lambda_{n} \sigma}{\lambda_{n}}=-\sigma<0 \quad \text { or } \quad \limsup _{n \rightarrow+\infty} \log A_{n}^{*} e^{-\lambda_{n} \sigma}=-\infty
$$

This implies that $\mu(\sigma, F)$ exists.

Many problems of analytic functions defined by Laplace-Stieltjes transformations have been studied and some important results have been obtained in [5-11]. In those papers, the authors mainly used the technique of a type function $U(x)$ to control the denominator in the definition of order. In 2012, Kong [12] investigated the growth of the Laplace-Stieltjes transforms convergent in the right half-plane by using a type function of the infinite order. In [13], the authors also investigated the growth and value distribution of infinite order analytic functions represented by Laplace-Stieltjes transformations convergent in the right half-plane. They introduced a completely new technique based on the concept of $X(x)$ to control the growth order of the numerator $\log M_{u}(\sigma, F)$ or $\log \mu(\sigma, F)$, and obtained the main theorems as follows. 
Theorem B (see [13]) If the Laplace-Stieltjes transformation $F(s)$ of infinite order has finite $X$-order, and sequence (2) satisfies (3) and (4), then we have

$$
\limsup _{\sigma \rightarrow 0^{+}} \frac{X\left(\log ^{+} M_{u}(\sigma, F)\right)}{\log \frac{1}{\sigma}}=\rho^{*} \quad \Longleftrightarrow \quad \limsup _{\sigma \rightarrow 0^{+}} \frac{X\left(\log ^{+} \mu(\sigma, F)\right)}{\log \frac{1}{\sigma}}=\rho^{*} .
$$

Theorem C (see [13]) If the Laplace-Stieltjes transformation $F(s)$ of infinite order and sequence (2) satisfies (3) and (4), then we have

$$
\limsup _{\sigma \rightarrow 0^{+}} \frac{X\left(\log ^{+} M_{u}(\sigma, F)\right)}{\log \frac{1}{\sigma}}=\rho^{\prime \prime} \Longleftrightarrow \limsup _{n \rightarrow \infty} \frac{X\left(\lambda_{n}\right)}{\log ^{+} \frac{\lambda_{n}}{\log ^{+} A_{n}^{*}}}=\rho^{\prime \prime},
$$

where $0<\rho^{*}<\infty$.

Remark 1.4 In Theorems B and C, the definitions of $X$-order and the function $X(x)$ are introduced in Section 2.

Thus, a question arises naturally: What will happen when $\rho^{\prime \prime}=\infty$ in Theorems $\mathrm{B}$ and C? In this paper, we investigate the above question by using the type functions $U(x)$ to enlarge the growth of the denominator $\log \frac{1}{\sigma}$, where $U(x)=x^{\rho(x)}$ satisfies the following conditions:

(i) $\rho(x)$ is monotone and $\lim _{x \rightarrow \infty} \rho(x)=\infty$;

(ii) $\lim _{x \rightarrow \infty} \frac{\log U\left(x^{\prime}\right)}{\log U(x)}=1$, where $x^{\prime}=x\left(1+\frac{1}{\log U(x)}\right)$.

Theorem 1.1 If the Laplace-Stieltjes transformation $F(s)$ of infinite order has infinite $X$-order, and sequence (2) satisfies (3) and (4), then we have

$$
\limsup _{\sigma \rightarrow 0^{+}} \frac{X\left(\log ^{+} M_{u}(\sigma, F)\right)}{\log U\left(\frac{1}{\sigma}\right)}=T \quad \Longleftrightarrow \quad \limsup _{\sigma \rightarrow 0^{+}} \frac{X\left(\log ^{+} \mu(\sigma, F)\right)}{\log U\left(\frac{1}{\sigma}\right)}=T,
$$

where $0<T<\infty$.

Remark 1.5 If the Laplace-Stieltjes transformation $F(s)$ of infinite order has infinite $X$-order and satisfies

$$
\limsup _{\sigma \rightarrow 0^{+}} \frac{X\left(\log M_{u}(\sigma, F)\right)}{\log U\left(\frac{1}{\sigma}\right)}=T,
$$

then $T$ is called the $X_{U}$-order of the Laplace-Stieltjes transform $F(s)$.

Remark 1.6 From Lemma 2.1 and Lemma 2.2 in Section 2, we can prove the conclusion of Theorem 1.1 easily.

Theorem 1.2 If the Laplace-Stieltjes transformation $F(s)$ has infinite X-order and sequence (2) satisfies (3) and (4), then we have

$$
\limsup _{\sigma \rightarrow 0^{+}} \frac{X\left(\log ^{+} M_{u}(\sigma, F)\right)}{\log U\left(\frac{1}{\sigma}\right)}=T \Longleftrightarrow \limsup _{n \rightarrow \infty} \frac{X\left(\lambda_{n}\right)}{\log ^{+} U\left(\frac{\lambda_{n}}{\log ^{+} A_{n}^{\sigma}}\right)}=T .
$$


From Theorem 1.2, we further investigate the value distribution of analytic functions with infinite $X$-order represented by Laplace-Stieltjes transformations convergent in the right half-plane and obtain the following theorems.

Theorem 1.3 Suppose that sequence (2) satisfies (3) and (4) and the Laplace-Stieltjes transformation $F(s)$ has infinite order. Let $\alpha(x)=\alpha_{1}(x)+i \alpha_{2}(x)$, where $\alpha_{1}(x)$ is an increasing function, and for any positive number $K>0$ and $|\delta|, \alpha_{2}(x)$ satisfies (5) and

$$
\left|\alpha_{2}(x+\delta)-\alpha_{2}(x)\right| \leq K\left|\alpha_{1}(x+\delta)-\alpha_{1}(x)\right|, \quad 0 \leq x, x+\delta<+\infty
$$

Then $s=0$ is the $X_{U}$-point of $F(s)$ with finite $X_{U}$-order $\varrho \geq T$, that is, for any $\eta>0$, the inequality

$$
\limsup _{\sigma \rightarrow 0^{+}} \frac{X(\bar{n}(\sigma, 0, \eta, F=a))}{\log U\left(\frac{1}{\sigma}\right)}=\varrho \geq T
$$

holds for any $a \in \mathbb{C}$ with one exception, where $\bar{n}(\sigma, 0, \eta, F=a)$ is the counting function of distinct zero of the function $F(s)-a$ in the strip $\{s: \Re(s)>\sigma,|\Im(s)|<\eta\}$.

Theorem 1.4 Suppose that sequence (2) satisfies (3) and (4), and the Laplace-Stieltjes transformation $F(s)$ is of infinite order. Let $\alpha(x)=\int_{0}^{x} r(y) e^{i t_{0} y} d y$, where $r(y)$ is a continuous function on $y \in[0,+\infty), r(y) \geq 0, t_{0}$ is a positive real number, and if $F(s)$ satisfies (5), then $s=i t_{0}$ is the $X_{U}$-point of $F(s)$ with finite $X_{U}$-order $\varrho \geq T$, that is, for any $\eta>0$, the inequality

$$
\limsup _{\sigma \rightarrow 0^{+}} \frac{X\left(\bar{n}\left(\sigma, i t_{0}, \eta, F=a\right)\right)}{\log U\left(\frac{1}{\sigma}\right)}=\varrho \geq T
$$

holds for any $a \in \mathbb{C}$ with one exception, where $\bar{n}\left(\sigma, i t_{0}, \eta, F=a\right)$ is the counting function of distinct zeros of the function $F(s)-a$ in the strip $\left\{s: \Re(s)>\sigma,\left|\Im(s)-t_{0}\right|<\eta\right\}$.

Theorem 1.5 Under the assumptions of Theorem 1.4, $l(\geq 1)$ is a positive integer. Then $s=0$ is the $X_{U}$-point dealing with multiple values of $F(s)$ with finite $X_{U}$-order $\varrho \geq T$, that is, for any $\eta>0$, the inequality

$$
\limsup _{\sigma \rightarrow 0^{+}} \frac{X\left(\bar{n}^{l)}(\sigma, 0, \eta, F=a)\right)}{\log U\left(\frac{1}{\sigma}\right)}=\varrho \geq T
$$

holds for any $a \in \mathbb{C}$ with at most $q\left(>1+\left[\frac{1}{l}\right]\right)$ exceptions, where $\bar{n}^{l)}(\sigma, 0, \eta, F=a)$ is the counting function of distinct zeros of the function $F(s)-a$ in the $\operatorname{strip}\{s: \Re(s)>\sigma,|\Im(s)|<\eta\}$, whose multiplicities are not greater than $l$.

Theorem 1.6 Under the assumptions of Theorem $1.4, l(\geq 1)$ is a positive integer. Then $s=i t_{0}$ is the $X_{U}$-point dealing with multiple values of $F(s)$ with finite $X_{U}$-order $\varrho \geq T$, that is, for any $\eta>0$, the inequality

$$
\limsup _{\sigma \rightarrow 0^{+}} \frac{X\left(\bar{n}^{l)}\left(\sigma, i t_{0}, \eta, F=a\right)\right)}{\log U\left(\frac{1}{\sigma}\right)}=\varrho \geq T
$$


holds for any $a \in \mathbb{C}$ with at most $q\left(>1+\left[\frac{1}{l}\right]\right)$ possible exceptions, where $\bar{n}^{l)}\left(\sigma, i t_{0}, \eta, F=a\right)$ is the counting function of distinct zeros of the function $F(s)-a$ in the $\operatorname{strip}\{s: \mathfrak{R}(s)>\sigma, \mid \Im(s)-$ $\left.t_{0} \mid<\eta\right\}$, whose multiplicities are not greater than $l$.

The structure of this paper is as follows. In Section 2, we introduce the concepts of $X$-order and $X_{U}$-order. Section 3 is devoted to proving Theorem 1.2. Section 4 is devoted to proving Theorems 1.3-1.6.

\section{The definitions of $X$-order and $X_{U}$-order}

We first introduce the concept of $X$-order of such functions as follows.

Definition 2.1 [14] If the Laplace-Stieltjes transform $F(s)$ satisfies $\sigma_{u}^{F}=0$ (sequence (2) satisfies (3) and (4)) and

$$
\limsup _{\sigma \rightarrow 0^{+}} \frac{\log ^{+} \log ^{+} M_{u}(\sigma, F)}{\log \frac{1}{\sigma}}=\infty
$$

then $F(s)$ is called a Laplace-Stieltjes transform of infinite order.

By studying a lot of papers, we found that to control the growth of the molecule $M_{u}(\sigma, F)$ or $\mu(\sigma, F)$ in the definition of order, many mathematicians proposed the type functions $U(x)$ to enlarge the growth of the denominator $\log \frac{1}{\sigma}$ or $-\sigma$ (see $\left.[4,6,7,10,11]\right)$. In this paper, we investigate the growth of the Laplace-Stieltjes transform of infinite order by using a class of functions to reduce the growth of $M_{u}(\sigma, F)$ or $\mu(\sigma, F)$ which is different from the previous form. Thus, we should give the definition of the new function as follows.

Let $\mathfrak{F}$ be the class of all functions $X(x)$ satisfying the following conditions:

(i) $X(x)$ is defined on $[a,+\infty), a>0$, is positive, strictly increasing, differential and tends to $+\infty$ as $x \rightarrow+\infty$;

(ii) $x X^{\prime}(x)=o(1)$ as $x \rightarrow+\infty$.

Definition 2.2 If the Laplace-Stieltjes transformation $F(s)$ of infinite order satisfies

$$
\limsup _{\sigma \rightarrow 0^{+}} \frac{X\left(\log M_{u}(\sigma, F)\right)}{\log \frac{1}{\sigma}}=\rho^{*},
$$

where $X(x) \in \mathfrak{F}$, then $\rho^{*}$ is called the $X$-order of the Laplace-Stieltjes transform $F(s)$.

Remark 2.1 In particular, if we take $X(x)=\log _{p} x, p \geq 2, p \in N_{+}$, where $\log _{1} x=\log x$ and $\log _{p} x=\log \left(\log _{p-1} x\right), X$-order is $p$-order of the Laplace-Stieltjes transformations with infinite order.

Remark 2.2 In addition, $X$-order is more precise than $p$-order to some extent. In fact, for $p(\geq 2)$ being a positive integer, we can find a function $X(x) \in \mathfrak{F}$ and a positive real function $M(x)$ satisfying

$$
\limsup _{x \rightarrow \infty} \frac{X(\log M(x))}{\log x}=A \quad(0<A<\infty)
$$


and

$$
\limsup _{x \rightarrow \infty} \frac{\log _{p}(\log M(x))}{\log x}=\infty \text { and } \quad \limsup _{x \rightarrow \infty} \frac{\log _{p+1}(\log M(x))}{\log x}=0 .
$$

For example, let $M(x)=\exp _{p+1}\left\{(t \log x)^{1 / d}\right\}, X(x)=\left(\log _{p} x\right)^{d}$, where $t$ is a finite positive real constant and $0<d<1$. We can get that $\rho_{p}(M)=\infty, \rho_{p+1}(M)=0$ and $\rho_{X}(M)=t$, where $\rho_{p}(f)$ denotes the $p$-order of $f$ and $\rho_{X}(f)$ the $X$-order of $f$.

Remark 2.3 If $\rho^{*}=\infty$ in Definition 2.1, then $F(s)$ is called a Laplace-Stieltjes transform of infinite $X$-order.

Lemma 2.1 Let $X(x) \in \mathfrak{F}$ and let $\beta(x)$ be the function satisfying

$$
\limsup _{x \rightarrow \infty} \frac{\log ^{+} \beta(x)}{\log x}=\varrho \quad(0 \leq \varrho<\infty) .
$$

If $M(x)$ satisfies $\lim \sup _{x \rightarrow \infty} \frac{X(\log M(x))}{\log x}=v(>0)$, then we have

$$
\limsup _{x \rightarrow \infty} \frac{X(\beta(x) \log M(x))}{\log x}=v
$$

Proof We consider two cases as follows.

Case 1. If $\beta(x)$ is not a constant. From the assumptions of Lemma 2.1, we can get that $\beta(x) \rightarrow \infty$ as $x \rightarrow \infty$. Thus, for sufficiently large $x$, we have $\beta(x)>1$. From $X(x) \in \mathfrak{F}$, we have $\lim _{x \rightarrow \infty} \log M(x)=\infty$. Then from the Cauchy mean value theorem, there exists $\xi(\log M(x)<\xi<\beta(x) \log M(x))$ satisfying

$$
\frac{X(\beta(x) \log M(x))-X(\log M(x))}{\log (\beta(x) \log M(x))-\log \log M(x)}=\frac{X^{\prime}(\xi)}{(\log \xi)^{\prime}}=\xi X^{\prime}(\xi),
$$

that is,

$$
X(\beta(x) \log M(x))=X(\log M(x))+\log \beta(x) \xi X^{\prime}(\xi)
$$

Since $x X^{\prime}(x)=o(1)$ as $x \rightarrow+\infty$ and $\limsup _{x \rightarrow \infty} \frac{\log \beta(x)}{\log x}=\varrho(0 \leq \varrho<\infty)$, by (6), we can get the conclusion of Lemma 2.1 easily.

Case 2. If $\beta(x)$ is a constant. By using the same argument as in Case 1 , we can prove the conclusion of Lemma 2.1 easily.

Thus, the conclusion of this lemma is true.

The following lemma is very crucial in the study of the growth of analytic functions represented by Laplace-Stieltjes transforms convergent in the right half-plane which show the relation between $M_{u}(\sigma, F)$ and $\mu(\sigma, F)$ of such functions.

Lemma $2.2[8,11]$ If the abscissa $\sigma_{u}^{F}=0$ of the uniformly convergent Laplace-Stieltjes transformation and the sequence (2) satisfies (3), then for any given $\varepsilon \in(0,1)$ and for $\sigma$ $(>0)$ sufficiently reaching 0 , we have

$$
\frac{1}{3} \mu(\sigma, F) \leq M_{u}(\sigma, F) \leq K(\varepsilon) \mu((1-\varepsilon) \sigma, F) \frac{1}{\sigma}
$$


where $K(\varepsilon)$ is a constant depending on $\varepsilon$, (3) and

$$
\log ^{+} x= \begin{cases}\log x, & x \geq 1 \\ 0, & x<1\end{cases}
$$

\section{The proof of Theorem 1.2}

We prove the conclusions of Theorem 1.2 by using the properties of two functions $X(x)$ and $U(x)$. This method is different from the previous method of [13] to some extent.

We first prove ' $\Longleftarrow$ ' of Theorem 1.2. Suppose that

$$
\limsup _{n \rightarrow \infty} \frac{X\left(\lambda_{n}\right)}{\log U\left(\frac{\lambda_{n}}{\log A_{n}^{\prime}}\right)}=T .
$$

Then, for any positive real number $\tau>0$, for sufficiently large $n$, we have

$$
\lambda_{n}<W\left((T+\tau) \log U\left(\frac{\lambda_{n}}{\log ^{+} A_{n}^{*}}\right)\right)
$$

where $W(x)$ is the inverse function of $X(x)$. Let $V(x)$ and $U(x)$ be two reciprocally inverse functions, then we have

$$
V\left(\exp \left\{\frac{1}{T+\tau} X\left(\lambda_{n}\right)\right\}\right)<\frac{\lambda_{n}}{\log ^{+} A_{n}^{* \prime}}, \quad \log ^{+} A_{n}^{*} \leq \lambda_{n}\left(V\left(\exp \left\{\frac{1}{T+\tau} X\left(\lambda_{n}\right)\right\}\right)\right)^{-1} .
$$

Thus, we have

$$
\log ^{+}\left(A_{n}^{*} e^{-\lambda_{n} \sigma}\right) \leq \lambda_{n}\left(\left(V\left(\exp \left\{\frac{1}{T+\tau} X\left(\lambda_{n}\right)\right\}\right)\right)^{-1}-\sigma\right) .
$$

For any fixed and sufficiently small $\sigma>0$, set

$$
G=W\left((T+\tau) \log U\left(\frac{1}{\sigma}+\frac{1}{\sigma \log U\left(\frac{1}{\sigma}\right)}\right)\right),
$$

that is,

$$
\frac{1}{\sigma}+\frac{1}{\sigma \log U\left(\frac{1}{\sigma}\right)}=V\left(\exp \left\{\frac{1}{T+\tau} X(G)\right\}\right) .
$$

If $\lambda_{n} \leq G$, for sufficiently large $n$, let $V\left(\exp \left\{\frac{1}{T+\tau} X\left(\lambda_{n}\right)\right\}\right) \geq 1$, from $\sigma>0$, (8), (9) and the definition of $U(x)$, we have

$$
\begin{aligned}
\log ^{+} A_{n}^{*} e^{-\lambda_{n} \sigma} & \leq G\left(\left(V\left(\exp \left\{\frac{1}{T+\tau} X\left(\lambda_{n}\right)\right\}\right)\right)^{-1}-\sigma\right) \\
& \leq G=W\left((T+\tau) \log U\left(\frac{1}{\sigma}+\frac{1}{\sigma \log U\left(\frac{1}{\sigma}\right)}\right)\right) \\
& \leq W\left((T+\tau) \log \left[(1+o(1)) U\left(\frac{1}{\sigma}\right)\right]\right) .
\end{aligned}
$$


If $\lambda_{n}>G$, from (8) and (9), we have

$$
\begin{aligned}
\log ^{+} A_{n}^{*} e^{-\lambda_{n} \sigma} & \leq \lambda_{n}\left(\left(V\left(\exp \left\{\frac{1}{T+\tau} X(G)\right\}\right)\right)^{-1}-\sigma\right) \\
& \leq \lambda_{n}\left(\left(\frac{1}{\sigma}+\frac{1}{\sigma \log U\left(\frac{1}{\sigma}\right)}\right)^{-1}-\sigma\right)<0
\end{aligned}
$$

For sufficiently large $n$, from (10) and (11), we have

$$
\log \mu(\sigma, F) \leq W\left((T+\tau) \log \left[(1+o(1)) U\left(\frac{1}{\sigma}\right)\right]\right)
$$

Since $\tau$ is arbitrary, by Theorem 1.1 and Lemma 2.1, we can get

$$
\limsup _{\sigma \rightarrow 0^{+}} \frac{X\left(\log M_{u}(\sigma, F)\right)}{\log U\left(\frac{1}{\sigma}\right)} \leq T
$$

Suppose that

$$
\limsup _{\sigma \rightarrow 0^{+}} \frac{X\left(\log M_{u}(\sigma, F)\right)}{\log U\left(\frac{1}{\sigma}\right)}<T
$$

Thus, there exists any real number $\varepsilon\left(0<\varepsilon<\frac{T}{2}\right)$. For any positive integer $n$ and any sufficiently small $\sigma>0$, from Lemma 2.2, we have

$$
\log ^{+} A_{n}^{*} e^{-\lambda_{n} \sigma} \leq \log M_{u}(\sigma, F) \leq W\left((T-2 \varepsilon) \log U\left(\frac{1}{\sigma}\right)\right)
$$

From (7), there exists a subsequence $\left\{\lambda_{n(p)}\right\}$; for sufficiently large $p$, we have

$$
X\left(\lambda_{n(p)}\right)>(T-\varepsilon) \log U\left(\frac{\lambda_{n(p)}}{\log ^{+} A_{n(p)}^{*}}\right)
$$

Take a sequence $\left\{\sigma_{p}\right\}$ satisfying

$$
W\left((T-2 \varepsilon) \log U\left(\frac{1}{\sigma_{p}}\right)\right)=\frac{\log ^{+} A_{n(p)}^{*}}{1+\log U\left(\frac{\lambda_{n(p)}}{\log ^{+} A_{n(p)}^{*}}\right)} .
$$

From (12) and (14), we get

$$
\log ^{+} A_{n(p)}^{*}-\lambda_{n(p)} \sigma_{p} \leq W\left((T-2 \varepsilon) \log U\left(\frac{1}{\sigma_{p}}\right)\right)=\frac{\log ^{+} A_{n(p)}^{*}}{1+\log U\left(\frac{\lambda_{n(p)}}{\log ^{+} A_{n(p)}^{*}}\right)}
$$

that is,

$$
\frac{1}{\sigma_{p}} \leq \frac{\lambda_{n(p)}}{\log ^{+} A_{n(p)}^{*}}\left(1+\frac{1}{\log U\left(\frac{\lambda_{n(p)}}{\log ^{+} A_{n(p)}^{*}}\right)}\right)
$$


Thus, we have

$$
U\left(\frac{1}{\sigma_{p}}\right) \leq U\left(\frac{\lambda_{n(p)}}{\log ^{+} A_{n(p)}^{*}}\left(1+\frac{1}{\log U\left(\frac{\lambda_{n(p)}}{\log ^{+} A_{n(p)}^{*}}\right)}\right)\right) \leq U^{1+o(1)}\left(\frac{\lambda_{n(p)}}{\log ^{+} A_{n(p)}^{*}}\right) .
$$

From (14) and (15), we have

$$
\begin{aligned}
\lambda_{n(p)} & =\frac{\lambda_{n(p)}}{\log ^{+} A_{n(p)}^{*}} W\left((T-2 \varepsilon) \log U\left(\frac{1}{\sigma_{p}}\right)\right)\left(1+\log U\left(\frac{\lambda_{n(p)}}{\log ^{+} A_{n(p)}^{*}}\right)\right) \\
& =\frac{\lambda_{n(p)}}{\log ^{+} A_{n(p)}^{*}} W\left((T-2 \varepsilon)(1+o(1)) \log U\left(\frac{\lambda_{n(p)}}{\log ^{+} A_{n(p)}^{*}}\right)\right)\left(1+\log U\left(\frac{\lambda_{n(p)}}{\log ^{+} A_{n(p)}^{*}}\right)\right) .
\end{aligned}
$$

Thus, from the Cauchy mean value theorem, there exists a real number $\xi$ between $\frac{\lambda_{n(p)}}{\log ^{+} A_{n(p)}^{*}}\left(1+\log U\left(\frac{\lambda_{n(p)}}{\log ^{+} A_{n(p)}^{*}}\right) W(T-2 \varepsilon)(1+o(1)) \log U\left(\frac{\lambda_{n(p)}}{\log ^{+} A_{n(p)}^{*}}\right)\right.$ and $W(T-2 \varepsilon)(1+o(1)) \times$ $\log U\left(\frac{\lambda_{n(p)}}{\log ^{+} A_{n(p)}^{* \prime}}\right)$ such that

$$
\begin{aligned}
X\left(\lambda_{n(p)}\right)= & X\left(\frac{\lambda_{n(p)}}{\log ^{+} A_{n(p)}^{*}}\left(1+\log U\left(\frac{\lambda_{n(p)}}{\log ^{+} A_{n(p)}^{*}}\right)\right)\right. \\
& \left.\times W\left((T-2 \varepsilon)(1+o(1)) \log U\left(\frac{\lambda_{n(p)}}{\log ^{+} A_{n(p)}^{*}}\right)\right)\right) \\
= & X\left(W\left((T-2 \varepsilon)(1+o(1)) \log U\left(\frac{\lambda_{n(p)}}{\log ^{+} A_{n(p)}^{*}}\right)\right)\right) \\
& +\log \left(\frac{\lambda_{n(p)}}{\log ^{+} A_{n(p)}^{*}}\left(1+\log U\left(\frac{\lambda_{n(p)}}{\log ^{+} A_{n(p)}^{*}}\right)\right)\right) \xi X^{\prime}(\xi) .
\end{aligned}
$$

Since

$$
\lim _{p \rightarrow \infty} \frac{\log \left(\frac{\lambda_{n(p)}}{\log ^{+} A_{n(p)}^{*}}\left(1+\log U\left(\frac{\lambda_{n(p)}}{\log ^{+} A_{n(p)}^{*}}\right)\right)\right)}{\log U\left(\frac{\lambda_{n(p)}}{\log ^{+} A_{n(p)}^{*}}\right)}=0
$$

then for sufficiently large $p$, we have

$$
X\left(\lambda_{n(p)}\right)=(T-2 \varepsilon)(1+o(1)) \log U\left(\frac{\lambda_{n(p)}}{\log ^{+} A_{n(p)}^{*}}\right)+K_{2} \xi X^{\prime}(\xi) \log U\left(\frac{\lambda_{n(p)}}{\log ^{+} A_{n(p)}^{*}}\right)
$$

where $K_{2}$ is a constant.

From (13) and (16), we can get a contradiction. Thus, we can get

$$
\underset{\sigma \rightarrow 0^{+}}{\limsup } \frac{X\left(\log M_{u}(\sigma, F)\right)}{\log U\left(\frac{1}{\sigma}\right)}=T
$$

Hence, the sufficiency of Theorem 1.2 is completed.

We can prove the necessity of Theorem 1.2 by using a similar argument as in the proof of sufficiency of Theorem 1.2.

Thus, the proof of Theorem 1.2 is completed. 


\section{Proofs of Theorems 1.3-1.6}

In this section, we give the definition of $X_{U}$-order of Laplace-Stieltjes transformations in the level half-strip as follows.

Definition 4.1 Let $F(s)$ be an analytic function with infinite $X$-order represented by Laplace-Stieltjes transformations convergent in the right half-plane. Set $S\left(t_{0}, l\right)=\{\sigma+i t$ : $\left.\sigma>0,\left|t-t_{0}\right| \leq l\right\}$, where $t_{0}$ is a real number and $l$ is a positive number. Let $X \in \mathfrak{F}$ and

$$
\tau_{S}^{X_{U}}=\limsup _{\sigma \rightarrow 0^{+}} \frac{X\left(\log ^{+} M_{S}(\sigma, F)\right)}{\log U\left(\frac{1}{\sigma}\right)}
$$

where $M_{S}(\sigma, F)=\sup _{\left|t-t_{0}\right| \leq l}|F(\sigma+i t)|$. Then $\tau_{S}^{X_{U}}$ is called the $X_{U}$-order of $F(S)$ in the level half-strip $S\left(t_{0}, l\right)$

To prove Theorems 1.3-1.6, we need some lemmas as follows.

Lemma 4.1 If the Laplace-Stieltjes transformation $F(s)$ is of infinite X-order, sequence (2) satisfies (3) and (4), and $\alpha(x)=\alpha_{1}(x)+i \alpha_{2}(x)$, where $\alpha_{1}(x)$ is an increasing function, and for any positive number $K>0$ and $|\delta|, \alpha_{2}(x)$ satisfies

$$
\left|\alpha_{2}(x+\delta)-\alpha_{2}(x)\right| \leq K\left|\alpha_{1}(x+\delta)-\alpha_{1}(x)\right|, \quad 0 \leq x, x+\delta<+\infty
$$

then for any $\varepsilon>0$, we have

$$
T=\limsup _{\sigma \rightarrow 0^{+}} \frac{X\left(\log ^{+} M_{u}(\sigma, F)\right)}{\log U\left(\frac{1}{\sigma}\right)}=\limsup _{\sigma \rightarrow 0^{+}} \frac{X\left(\log ^{+} M_{S_{\varepsilon}}(\sigma, F)\right)}{\log U\left(\frac{1}{\sigma}\right)}=\tau_{S_{\varepsilon}}^{X_{U}} .
$$

Proof We will prove this lemma by using a similar argument to that in [11]. From the assumptions of Lemma 4.1, for any $0<x \leq \infty$,

$$
\begin{aligned}
M_{S_{\varepsilon}}(\sigma, F) & \geq\left|\int_{0}^{\infty} e^{-\sigma y} d \alpha(y)\right| \geq \int_{0}^{\infty} e^{-\sigma y} d \alpha_{1}(y) \\
& \geq \frac{1}{K+1} \int_{0}^{\infty} e^{-\sigma y}\left|d \alpha_{1}(y)\right| \geq \frac{1}{K+1} \int_{0}^{x} e^{-\sigma y}\left|d \alpha_{1}(y)\right| \\
& \geq \frac{1}{K+1}\left|\int_{0}^{\infty} e^{-(\sigma+i t) y} d \alpha(y)\right|
\end{aligned}
$$

Then

$$
M(\sigma, F) \geq M_{S_{\varepsilon}}(\sigma, F) \geq \frac{1}{K+1} M_{u}(\sigma, F) \geq \frac{1}{K+1} M(\sigma, F) .
$$

Since $F(s)$ is an analytic function with infinite $X$-order, from the above inequality and the definition of $X_{U}$-order, we can get the conclusion of Lemma 4.1.

Lemma 4.2 [11, Lemma 2.4] Let

$$
z=\frac{1-\sinh s}{1+\sinh s}, \quad s \in B=\left\{s: \Re(s)>0,|\Im(s)|<\frac{\pi}{2}\right\} .
$$


Then

(i) this mapping maps the horizontal half-strip B to the unit disc $\{z:|z|<1\}$, and its inverse mapping is

$$
s=\Psi(z)=\sinh ^{-1} \frac{1-z}{1+z}
$$

(ii) $\min _{0 \leq \theta \leq 2 \pi} \Re\left[\Psi\left(r e^{i \theta}\right)\right] \geq \Psi(r)(0<r<1)$;

(iii) $\max _{0 \leq \theta \leq \frac{\pi}{4}} \Re\left[\Psi\left(r e^{i \theta}\right)\right] \leq \Psi\left(r^{2}\right)(0<r<1)$;

(iv) $\Psi(\{z:|z|<r\}) \subseteq\left\{s: \Re(s)>\Psi(r),|\Im(s)|<\frac{\pi}{2}\right\}(0<r<1)$.

Lemma 4.3 (see [14]) Let $f$ be an admissible function in the unit disc $\mathbb{D}$, let $q$ be a positive integer, and let $a_{1}, \ldots, a_{q}$ be pairwise distinct complex numbers. Then, for $r \rightarrow 1^{-}, r \notin E$,

$$
(q-2) T(r, f) \leq \sum_{j=1}^{q} \bar{N}\left(r, \frac{1}{f-a_{j}}\right)+S(r, f)
$$

where $E \subset(0,1)$ is a possibly occurring exceptional set with $\int_{E} \frac{d r}{1-r}<\infty$, and the term $\bar{N}\left(r, \frac{1}{f-a_{j}}\right)$ is replaced by $\bar{N}(r, f)$ when some $a_{j}=\infty$. We use $S(r, f)$ to denote

$$
S(r, f)=O\left\{\log \frac{1}{1-r}\right\}+O\left\{\log ^{+} T(r, f)\right\}
$$

as $r \rightarrow 1^{-}$possibly outside the set $E$ such that $\int_{E} \frac{d r}{1-r}<\infty$. If the order of $f$ is finite, the remainder $S(r, f)$ is an $O\left(\log \frac{1}{1-r}\right)$ without any exceptional set.

Remark 4.1 Under the assumptions of Lemma 4.3, for a positive integer $l$, we can get the following inequality easily:

$$
\left(q-2-\frac{2}{l}\right) T(r, f) \leq \sum_{j=1}^{q} \bar{N}^{l}\left(r, \frac{1}{f-a_{j}}\right)+S(r, f)
$$

where $\bar{N}^{l)}\left(r, \frac{1}{f-a_{i}}\right)$ is the counting function of poles of the function $\frac{1}{f-a_{j}}$ with multiplicities $\leq l$ in $\{z:|z| \leq r\}$, each point counted only once.

Lemma 4.4 [15, p.282, (1.8)] Let $h$ be an analytic in the disc $|z|=r<1$, then

$$
T(r, h) \leq \log M(r, h) \leq \frac{1+r}{1-r} T(r, h)
$$

where $M(r, h)$ is the maximum modulus of $h$ in the disc $|z|=r<1$.

\subsection{The proof of Theorem 1.3}

Since sequence (2) satisfies (3) and (4), the Laplace-Stieltjes transformation $F(s)$ of infinite $X$-order, and $\lim \sup _{n \rightarrow \infty} \frac{X\left(\lambda_{n}\right)}{\log U\left(\frac{\lambda_{n}}{\left.\log ^{+} A_{n}\right)}\right.}=T(0<T<\infty)$, from Theorem 1.2, we have

$$
\limsup _{\sigma \rightarrow 0^{+}} \frac{X\left(\log ^{+} M_{u}(\sigma, F)\right)}{\log U\left(\frac{1}{\sigma}\right)}=T
$$


and from Lemma 4.1 and (17), for any $\varepsilon>0$, we have

$$
\limsup _{\sigma \rightarrow 0^{+}} \frac{X\left(\log ^{+} M_{S_{\varepsilon}}(\sigma, F)\right)}{\log U\left(\frac{1}{\sigma}\right)}=T
$$

Thus, it follows

$$
\limsup _{\sigma \rightarrow 0^{+}} \frac{X\left(\log M\left(\sigma, F, S_{\varepsilon}\right)\right)}{\log U\left(\frac{1}{\sigma}\right)}=T,
$$

where $S_{\varepsilon}=\{s: \Re(s)>0,|\Im(s)| \leq \varepsilon\}$ and $M\left(\sigma, F, S_{\varepsilon}\right)=\sup \left\{|F(s)|: \Re(s) \geq \sigma, s \in S_{\varepsilon}\right\}$.

Set $g(z)=F\left(\frac{2 \varepsilon}{\pi} \Psi(z)\right)$, where $\Psi(z)$ is stated as in Lemma 4.2. Then from Lemma 4.2, we get that $g(z)$ is analytic in the unit disc $|z|<1$ and satisfies

$$
M\left(\frac{2 \varepsilon}{\pi} \Psi\left(r^{2}\right), F, S_{\varepsilon_{1}}\right) \leq M(r, g) \leq M\left(\frac{2 \varepsilon}{\pi} \Psi(r), F, S_{\varepsilon}\right),
$$

where $0<\varepsilon_{1}<\varepsilon$. Therefore, from (18), (19) and Lemma 2.1, we have

$$
\limsup _{r \rightarrow 1^{-}} \frac{X\left(\log ^{+} M(r, g)\right)}{\log \frac{1}{1-r}}=\infty, \quad \limsup _{r \rightarrow 1^{-}} \frac{X\left(\log ^{+} M(r, g)\right)}{\log U\left(\frac{1}{1-r}\right)}=T .
$$

From (20), Lemma 4.4 and Lemma 2.1, we can get that $g$ is an admissible function in $|z|<1$ and

$$
\limsup _{r \rightarrow 1^{-}} \frac{X(T(r, g))}{\log U\left(\frac{1}{1-r}\right)}=T
$$

Then from Lemma 4.3 and (21), we can get that at most there exists one exception $a$ satisfying

$$
\limsup _{r \rightarrow 1^{-}} \frac{X(\bar{N}(r, g=a))}{\log U\left(\frac{1}{1-r}\right)}=\varrho \geq T .
$$

Since

$$
\bar{n}(r, g=a) \log \frac{1+r}{2 r} \leq \int_{r}^{\frac{1+r}{2}} \frac{\bar{n}(t, g=a)}{t} d t \leq \bar{N}\left(\frac{1+r}{2}, g=a\right) \log \frac{1+r}{2 r}
$$

and

$$
\bar{N}(r, g=a)-\bar{N}\left(r_{0}, g=a\right) \leq \int_{r_{0}}^{r} \frac{\bar{n}(t, g=a)}{t} d t \leq \bar{n}(r, g=a) \log \frac{r}{r_{0}}, \quad r_{0}<r<1,
$$

we have

$$
\bar{n}(r, g=a) \leq \bar{N}\left(\frac{1+r}{2}, g=a\right) \leq \bar{n}(r, g=a) \log \frac{r}{r_{0}}+O(1), \quad r_{0}<r<1
$$

Thus, from (22)-(23) and Lemma 2.1, we have

$$
\limsup _{r \rightarrow 1^{-}} \frac{X(\bar{n}(r, g=a))}{\log U\left(\frac{1}{1-r}\right)}=\varrho \geq T
$$


Hence, for any $\eta>0$ and (24), the inequality

$$
\limsup _{\sigma \rightarrow 0^{+}} \frac{X(\bar{n}(\sigma, 0, \eta, F=a))}{\log U\left(\frac{1}{\sigma}\right)}=\varrho \geq T
$$

holds for any $a \in \mathbb{C}$ with one exception, where $\bar{n}(\sigma, 0, \eta, F=a)$ is the counting function of zeros of the function $F(s)-a$ in the strip $\{s: \mathfrak{R}(s)>\sigma,|\Im(s)|<\eta\}$.

Thus, we complete the proof of Theorem 1.3.

\subsection{The proof of Theorem 1.4}

Since $r(y)$ is a continuous function on $y \in[0,+\infty)$, then we can get that $\alpha(x)=\int_{0}^{x} r(y) e^{i t_{0} y} d y$ is a function of bounded variation on $x \in[0, Y](0<Y<\infty)$. Set

$$
\widehat{S}_{\varepsilon}=\left\{s: \Re(s)>0,\left|\Im(s)-t_{0}\right| \leq \varepsilon\right\}, \quad M_{\widehat{S}_{\varepsilon}}(\sigma, F)=\sup _{\left|t-t_{0}\right| \leq \varepsilon}|F(\sigma+i t)| .
$$

From the assumptions of Theorem 1.6, for any real number $x(0<x \leq \infty)$, we have

$$
\begin{aligned}
M_{\widehat{S}_{\varepsilon}}(\sigma, F) & =\sup _{\left|t-t_{0}\right| \leq \varepsilon}|F(\sigma+i t)|=\sup _{\left|t-t_{0}\right| \leq \varepsilon}\left|\int_{0}^{\infty} e^{-s y} r(y) e^{i t_{0} y} d y\right| \\
& \geq \int_{0}^{\infty} e^{-\sigma y} r(y) d y \geq \int_{0}^{x} e^{-\sigma y} r(y) d y \\
& \geq\left|\int_{0}^{x} e^{-s y} r(y) e^{i t_{0} y} d y\right|=\left|\int_{0}^{\infty} e^{-s y} d \alpha(y)\right|,
\end{aligned}
$$

that is,

$$
M(\sigma, F) \leq M_{u}(\sigma, F) \leq M_{\widehat{S}_{\varepsilon}}(\sigma, F) \leq M(\sigma, F)
$$

From the assumption of Theorem 1.4, by (25) and Theorem 1.1, we can get

$$
\begin{aligned}
\underset{\sigma \rightarrow 0^{+}}{\limsup \frac{X\left(\log ^{+} M_{u}(\sigma, F)\right)}{\log U\left(\frac{1}{\sigma}\right)}} & =\underset{\sigma \rightarrow 0^{+}}{\limsup } \frac{X\left(\log ^{+} M_{\widehat{S}_{\varepsilon}}(\sigma, F)\right)}{\log U\left(\frac{1}{\sigma}\right)} \\
& =\underset{\sigma \rightarrow 0^{+}}{\limsup } \frac{X\left(\log ^{+} M(\sigma, F)\right)}{\log U\left(\frac{1}{\sigma}\right)}=T .
\end{aligned}
$$

From (26), for any $\varepsilon>0$, we have

$$
\limsup _{\sigma \rightarrow 0^{+}} \frac{X\left(\log ^{+} M\left(\sigma, F, \widehat{S}_{\varepsilon}\right)\right)}{\log U\left(\frac{1}{\sigma}\right)}=T,
$$

where $M\left(\sigma, F, \widehat{S}_{\varepsilon}\right)=\sup \left\{|F(s)|: \Re(s) \geq \sigma, s \in \widehat{S}_{\varepsilon}\right\}$.

Set $g(z)=F\left(\frac{2 \varepsilon}{\pi} \Psi(z)+i t_{0}\right)$, where $\Psi(z)$ is stated as in Lemma 4.2. Then from Lemma 4.2, we can get that $g(z)$ is analytic in the unit disc $|z|<1$ and satisfies

$$
M\left(\frac{2 \varepsilon}{\pi} \Psi\left(r^{2}\right), F, S_{\varepsilon_{2}}\right) \leq M(r, g) \leq M\left(\frac{2 \varepsilon}{\pi} \Psi(r), F, S_{\varepsilon}\right),
$$


where $0<\varepsilon_{2}<\varepsilon$. Therefore, from the above inequality and Lemma 4.2, we have

$$
\limsup _{r \rightarrow 1^{-}} \frac{X\left(\log ^{+} M(r, g)\right)}{\log \frac{1}{1-r}}=\infty, \quad \limsup _{r \rightarrow 1^{-}} \frac{X\left(\log ^{+} M(r, g)\right)}{\log U\left(\frac{1}{1-r}\right)}=T .
$$

Then, similar to the proof of Theorem 1.3, we can prove that for any $\eta>0$, the inequality

$$
\limsup _{\sigma \rightarrow 0^{+}} \frac{X\left(\bar{n}\left(\sigma, i t_{0}, \eta, F=a\right)\right)}{\log \frac{1}{\sigma}}=\varrho \geq \rho^{*}
$$

holds for any $a \in \mathbb{C}$ with one exception, where $\bar{n}\left(\sigma, i t_{0}, \eta, F=a\right)$ is stated as in Theorem 1.6.

Thus, we complete the proof of Theorem 1.4.

\subsection{Proofs of Theorems 1.5 and 1.6}

From Remark 4.1, by using the same argument as in Theorems 1.3 and 1.4, we can prove the conclusions of Theorems 1.5 and 1.6 easily.

\section{Competing interests}

The authors declare that they have no competing interests.

\section{Authors' contributions}

HYX and ZXX completed the main part of this article, ZXX corrected the main theorems. All authors read and approved the final manuscript.

\section{Author details}

${ }^{1}$ Department of Informatics and Engineering, Jingdezhen Ceramic Institute, Jingdezhen, Jiangxi 333403, China. ${ }^{2}$ Beijing Key Laboratory of Information Service Engineering, Department of General Education, Beijing Union University, No. 97 Bei Si Huan Dong Road, Chaoyang District, Beijing, 100101, China.

\section{Acknowledgements}

This project is supported by the NSFC (No. 61202313, 11201395) and the Natural Science foundation of Jiangxi Province in China (No. 2010GQS0119, No. 20122BAB201016 and No. 20132BAB211001). The second author is supported in part by NNSFC (Nos. 11226089, 61271370), Beijing Natural Science Foundation (No. 1132013) and The Project of Construction of Innovative Teams and Teacher Career Development for Universities and Colleges Under Beijing Municipality (CIT\&TCD20130513)

Received: 10 November 2012 Accepted: 10 April 2013 Published: 31 May 2013

\section{References}

1. Liu, MS: The regular growth of Dirichlet series of finite order in the half plane. J. Syst. Sci. Math. Sci. 22(2), 229-238 (2002)

2. Sun, DC: On the distribution of values of random Dirichlet series (II). Chin. Ann. Math., Ser. B 11(1), 33-44 (1990)

3. Yu, JR: Dirichlet Series and the Random Dirichlet Series. Science Press, Beijing (1997)

4. Yu, JR: Borel's line of entire functions represented by Laplace-Stieltjes transformation. Acta Math. Sin. 13, 471-484 (1963) (in Chinese)

5. Batty, CJK: Tauberian theorem for the Laplace-Stieltjes transform. Trans. Am. Math. Soc. 322(2), $783-804$ (1990)

6. Kong, YY, Sun, DC: On the growth of zero order Laplace-Stieltjes transform convergent in the right half-plane. Acta Math. Sci., Ser. B 28(2), 431-440 (2008)

7. Kong, YY, Sun, DC: On type-function and the growth of Laplace-Stieltjes transformations convergent in the right half-plane. Adv. Math. 37(2), 197-205 (2007) (in Chinese)

8. Kong, YY, Sun, DC: The analytic function in the right half plane defined by Laplace-Stieltjes transforms. J. Math. Res. Expo. 28(2), 353-358 (2008)

9. Mishkelyavichyus, A: A Tauberian theorem for the Laplace-Stieltjes integral and the Dirichlet series. Litov. Mat. Sb. 29(4), 745-753 (1989) (Russian)

10. Shang, LN, Gao, ZS: The growth of entire functions of infinite order represented by Laplace-Stieltjes transformation Acta Math. Sci., Ser. A 27(6), 1035-1043 (2007) (in Chinese)

11. Shang, LN, Gao, ZS: The value distribution of analytic functions defined by Laplace-Stieltjes transforms. Acta Math Sin. Chin. Ser. 51(5), 993-1000 (2008)

12. Kong, YY: Laplace-Stieltjes transforms of infinite order in the right half-plane. Acta Math. Sin. Chin. Ser. 55(1), 141-148 (2012)

13. $\mathrm{Xu}, \mathrm{HY}, \mathrm{Xuan}, \mathrm{ZX}$ : The value distribution of analytic functions with infinite order defined by Laplace-Stieltjes transformations convergent on the right half-plane (submitted)

14. Hayman, WK: Meromorphic Functions. Oxford University Press, London (1964)

15. $\mathrm{CaO}, \mathrm{TB}, \mathrm{Yi}, \mathrm{HX}$ : The growth of solutions of linear differential equations with coefficients of iterated order in the unit disc. J. Math. Anal. Appl. 319, 278-294 (2006) 
doi:10.1186/1029-242X-2013-273

Cite this article as: $\mathrm{Xu}$ and Xuan: The growth and value distribution of Laplace-Stieltjes transformations with infinite order in the right half-plane. Journal of Inequalities and Applications 2013 2013:273.

Submit your manuscript to a SpringerOpen ${ }^{\circ}$ journal and benefit from:

- Convenient online submission

- Rigorous peer review

- Immediate publication on acceptance

- Open access: articles freely available online

- High visibility within the field

- Retaining the copyright to your article

Submit your next manuscript at $\gg$ springeropen.com 